MATHEMATICS OF COMPUTATION

Volume 76, Number 258, April 2007, Pages 631-646

S 0025-5718(06)01938-7

Article electronically published on December 27, 2006

\title{
MAXIMUM NORM ERROR ANALYSIS OF A 2D SINGULARLY PERTURBED SEMILINEAR REACTION-DIFFUSION PROBLEM
}

\author{
NATALIA KOPTEVA
}

Dedicated to Professor V. B. Andreev on the occasion of his 65th birthday

\begin{abstract}
A semilinear reaction-diffusion equation with multiple solutions is considered in a smooth two-dimensional domain. Its diffusion parameter $\varepsilon^{2}$ is arbitrarily small, which induces boundary layers. Constructing discrete sub- and super-solutions, we prove existence and investigate the accuracy of multiple discrete solutions on layer-adapted meshes of Bakhvalov and Shishkin types. It is shown that one gets second-order convergence (with, in the case of the Shishkin mesh, a logarithmic factor) in the discrete maximum norm, uniformly in $\varepsilon$ for $\varepsilon \leq C h$. Here $h>0$ is the maximum side length of mesh elements, while the number of mesh nodes does not exceed $C h^{-2}$. Numerical experiments are performed to support the theoretical results.
\end{abstract}

\section{INTRODUCTION}

Consider the singularly perturbed semilinear reaction-diffusion boundary-value problem

$$
\begin{aligned}
F u \equiv-\varepsilon^{2} \triangle u+b(x, u)=0, & x=\left(x_{1}, x_{2}\right) \in \Omega \subset \mathbb{R}^{2}, \\
u(x)=g(x), & x \in \partial \Omega,
\end{aligned}
$$

where $\varepsilon$ is a small positive parameter, $\triangle=\partial^{2} / \partial x_{1}^{2}+\partial^{2} / \partial x_{2}^{2}$ is the Laplace operator, and $\Omega$ is a bounded two-dimensional domain whose boundary $\partial \Omega$ is sufficiently smooth. Assume also that the functions $b$ and $g$ are sufficiently smooth. We shall examine solutions of (1.1) that exhibit boundary layer behaviour. In general, solutions of (1.1) may also have interior transition layers, which we will consider in a future paper.

The reduced problem of (1.1) is defined by formally setting $\varepsilon=0$ in (1.1a), viz.,

$$
b\left(x, u_{0}(x)\right)=0 \quad \text { for } x \in \Omega .
$$

Note that any solution $u_{0}$ of (1.2) does not in general satisfy the boundary condition in $(1.1 \mathrm{~b})$.

Received by the editor October 8, 2005 and, in revised form, February 23, 2006.

2000 Mathematics Subject Classification. Primary 65N06, 65N15, 65N30; Secondary 35B25.

Key words and phrases. Semilinear reaction-diffusion, singular perturbation, maximum norm error estimate, $Z$-field, Bakhvalov mesh, Shishkin mesh, second order.

This publication has emanated from research conducted with the financial support of Science Foundation Ireland under the Basic Research Grant Programme 2004; Grant 04/BR/M0055.

(C)2006 American Mathematical Society Reverts to public domain 28 years from publication 

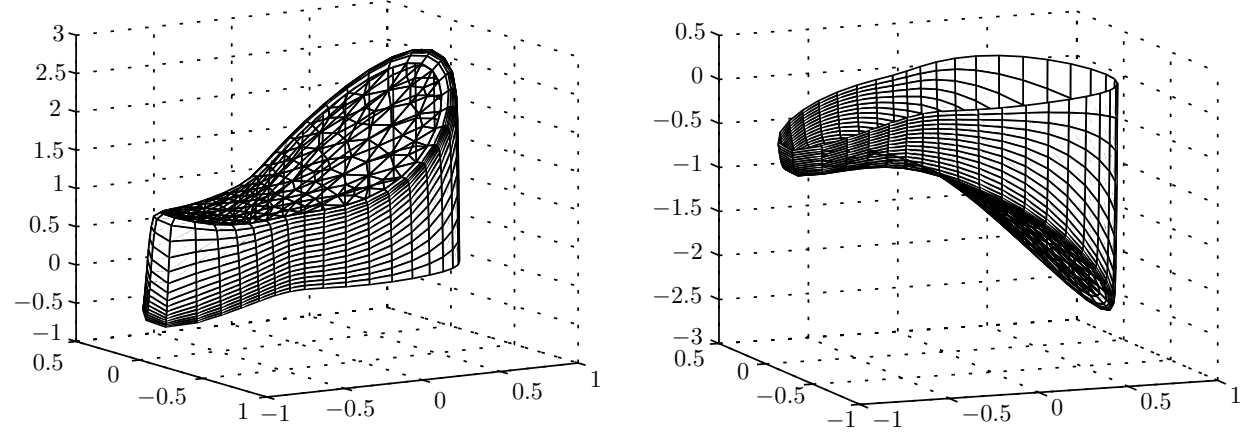

Figure 1. Multiple boundary-layer solutions of model problem (4.1); in the interior subdomain $u(x) \approx \bar{u}_{0}(x)$ (left) or $u(x) \approx$ $-\bar{u}_{0}(x)$ (right), where $\pm \bar{u}_{0}(x)$ are stable solutions of the reduced problem (1.2).

In the numerical analysis literature it is often assumed (see, e.g., 15, 2, that $b_{u}(x, u)>\gamma^{2}>0$ for all $(x, u) \in \Omega \times \mathbb{R}^{1}$, for some positive constant $\gamma$. Under this condition the reduced problem has a unique solution $u_{0}$ that is sufficiently smooth in $\bar{\Omega}$, as can be seen by using the implicit function theorem and the compactness of $\bar{\Omega}$. This global condition is nevertheless rather restrictive. E.g., mathematical models of biological and chemical processes frequently involve problems related to (1.1) with $b(x, u)$ that is non-monotone with respect to $u$ [11, §14.7], [7, §2.3]. Hence we consider problem (1.1) under the following weaker assumptions from [6, 12]:

- it has a stable reduced solution, i.e., there exists a sufficiently smooth solution $u_{0}$ of (1.2) such that

$$
b_{u}\left(x, u_{0}\right)>\gamma^{2}>0 \text { for all } x \in \Omega
$$

- the boundary condition satisfies

$$
\int_{u_{0}(x)}^{v} b(x, s) d s>0 \quad \text { for all } v \in\left(u_{0}(x), g(x)\right]^{\prime}, \quad x \in \partial \Omega .
$$

Here the notation $(a, b]^{\prime}$ is defined to be $(a, b]$ when $a<b$ and $[b, a)$ when $a>b$, while $(a, b]^{\prime}=\emptyset$ when $a=b$.

Note that if $g(x) \approx u_{0}(x)$, then (A2) follows from (A1) combined with (1.2), while if $g(x)=u_{0}(x)$ at some point $x \in \partial \Omega$, then (A2) does not impose any restriction on $g$ at this point.

Conditions (A1), (A2) intrinsically arise from the asymptotic analysis of problem (1.1) and guarantee that there exists a boundary-layer solution $u$ of (1.1) such that $u \approx u_{0}$ in the interior subdomain of $\Omega$ away from the boundary, while the boundary layer is of width $O(\varepsilon|\ln \varepsilon|)$ [6, 12]; see Theorem 2.2 for a precise statement and [8] for a detailed discussion of (A1), (A2) in one dimension. Note that assumption (A1) is local, i.e., the reduced problem (1.2) is permitted to have more than one stable solution. Furthermore, if multiple stable solutions of the reduced problem satisfy (A2), problem (1.1) has multiple boundary-layer solutions; see Figure 1

We discretize the domain as in Figure 2 (see $\$ 3.1$ for details) using layer-adapted meshes of Bakhvalov and Shishkin types whose number of mesh nodes does not 


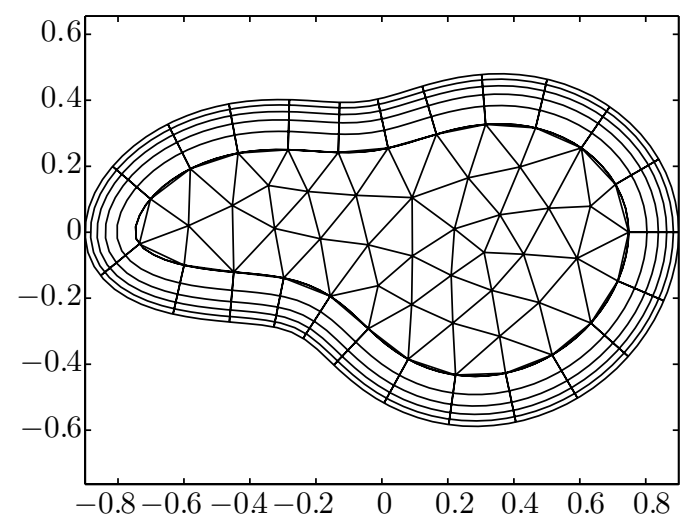

FiguRE 2. Layer-adapted mesh.

exceed $C h^{-2}$. Here $h>0$ is the maximum side length of mesh elements of the layer-adapted meshes that we consider.

Then we discretize equation (1.1a) combining finite differences on the curvilinear tensor-product part of the mesh and lumped mass linear finite elements on a quasiuniform Delaunay triangulation in the interior region. The key feature of our method is that it uses an $M$-matrix discretization of the operator $-\triangle$.

Constructing discrete sub- and super-solutions, we prove existence and investigate the accuracy of multiple discrete solutions of problem (1.1). Our main result is Theorem 3.20 that states second-order convergence (with, in the case of the Shishkin mesh, a logarithmic factor) in the discrete maximum norm, uniformly in $\varepsilon$.

We make two further simplifying assumptions to facilitate our presentation. To avoid considering cases, assume that

$$
u_{0}(x)<g(x) \text { for all } x \in \partial \Omega .
$$

Throughout our analysis take

$$
\varepsilon \leq C h
$$

This is not a practical restriction, and from a theoretical viewpoint the analysis of a non-linear problem such as (1.1) would be very different if $\varepsilon$ were not small.

A one-dimensional version of problem (1.1) was studied in [5, 17, 8. The present paper extends the analysis [8 to two dimensions. Linear analogues of the twodimensional problem (1.1) were considered in [10, 4. Melenk [10] gives energy norm error estimates for $h p$-finite element methods on layer-adapted meshes in a curvilinear polygon. Clavero et al. 4] present maximum norm error estimates for finite differences on Shishkin meshes in the unit square. The semilinear problem (1.1) under the condition $b_{u}>\gamma^{2}>0$ for all $(x, u)$ was considered in [15, 2, 3]. Schatz and Wahlbin [15] derive pointwise error estimates for the Galerkin finite elements on quasiuniform unrefined meshes in polygonal domains. Blatov [2, 3, proves second-order convergence in the discrete maximum norm of a finite element method on a Bakhvalov-type mesh in a smooth domain (under the condition $\varepsilon|\ln \varepsilon| \leq C h$ similar to (A4)). Note that the meshes that are considered in the present paper resemble the mesh [2, 3, while our numerical method is different. In 2, 3] bilinear 
elements are used on the curvilinear tensor-product part of the mesh. Furthermore, our analysis is completely different from the analysis by Blatov. To be more precise, Blatov estimates the error in terms of the interpolation error, which involves rather technical arguments, while we obtain error bounds using modified asymptotic expansions for problem (1.1) and then invoking the theory of $Z$-fields.

The paper is organized as follows. In $₫ 2$ we discuss asymptotic properties of solutions of (1.1) and construct sub- and super-solutions. In 93 layer-adapted meshes and a numerical method for solving (1.1) are described, and discrete analogues of the sub- and super-solutions are used to obtain tight upper and lower bounds on the computed solutions. Precise convergence results for the numerical method are then derived on Bakhvalov and Shishkin meshes. Finally, in 4 , numerical results illustrate the sharpness of our convergence bounds.

Notation. Throughout this paper we let $C$ denote a generic positive constant that may take different values in different formulas, but is always independent of $h$ and $\varepsilon$. A subscripted $C$ (e.g., $C_{1}$ ) denotes a positive constant that is independent of $h$ and $\varepsilon$ and takes a fixed value. For any two quantities $w_{1}$ and $w_{2}$, the notation $w_{1}=O\left(w_{2}\right)$ means $\left|w_{1}\right| \leq C w_{2}$. For any function $g \in C(\bar{\Omega})$, the notation $g_{i}$ or $g_{i j}$ means $g\left(X_{i}\right)$ or $g\left(x_{i j}\right)$, respectively, where $X_{i} \in \bar{\Omega}$ and $x_{i j} \in \bar{\Omega}$ are mesh nodes.

\section{Asymptotic AnAlysis, SUB- AND SUPER-SOlUtions}

We start this section by sketching the asymptotic expansion from [12]; see also [19, §4], [18, §3.1.1] for the linear case. Furthermore, we modify it to construct certain sub- and super-solutions that provide tight control on the solutions of (1.1).

2.1. Local curvilinear coordinates. Let the boundary $\partial \Omega$ be parametrized by

$$
x_{1}=\varphi(l), \quad x_{2}=\psi(l), \quad 0 \leq l \leq L,
$$

where $(\varphi(0), \psi(0))=(\varphi(L), \psi(L))$ and as $l$ increases, the domain remains on the left. Any functions that are defined for $l$ beyond $[0, L]$ should be understood as extended $L$-periodically. We shall use the magnitude $\tau>0$ of the tangent vector $\left(\varphi^{\prime}, \psi^{\prime}\right)$ and the curvature $\kappa$ of the boundary at $(\varphi(l), \psi(l))$ that are defined by

$$
\tau=\sqrt{\varphi^{\prime 2}+\psi^{\prime 2}}, \quad \kappa=\kappa(l)=\frac{\varphi^{\prime} \psi^{\prime \prime}-\psi^{\prime} \varphi^{\prime \prime}}{\tau^{3}} .
$$

In a narrow neighbourhood of $\partial \Omega$ that will be specified later, introduce the curvilinear local coordinates $(r, l)$ by

$$
x_{1}=\varphi(l)+r n_{1}(l), \quad x_{2}=\psi(l)+r n_{2}(l),
$$

where $\left(n_{1}, n_{2}\right)$ is the inward unit normal to $\partial \Omega$ at $(\varphi(l), \psi(l))$, i.e., it is orthogonal to the tangent vector $\left(\varphi^{\prime}, \psi^{\prime}\right)$ and is defined by

$$
n_{1}=\frac{-\psi^{\prime}}{\tau}, \quad n_{2}=\frac{\varphi^{\prime}}{\tau}
$$

Since $\partial \Omega$ is smooth, there exists a sufficiently small constant $C_{1}$ such that in the subdomain $\bar{\Omega}_{C_{1}}=\left\{0 \leq r \leq C_{1}\right\}$ the new coordinates are well-defined and the mapping $(r, l) \mapsto\left(x_{1}, x_{2}\right)$ is one-to-one and invertible. Throughout the paper we shall use a smooth positive cut-off function $\omega(x)$ that equals 1 for $r \leq C_{1} / 2$ and vanishes in $\bar{\Omega} \backslash \bar{\Omega}_{C_{1}}$. 
Lemma 2.1. The curvilinear coordinates (2.1) are orthogonal, and for the Laplace operator we have

$$
\triangle u=\eta^{-1} \frac{\partial}{\partial r}\left(\eta \frac{\partial u}{\partial r}\right)+\zeta \frac{\partial}{\partial l}\left(\zeta \frac{\partial u}{\partial l}\right), \quad \text { where } \eta:=1-\kappa r, \zeta:=(\tau \eta)^{-1} .
$$

We defer the proof of this lemma to Appendix $\mathrm{A}$

2.2. First-order asymptotic expansion. To construct an asymptotic expansion, introduce the stretched variable

$$
\xi:=r / \varepsilon
$$

and the functions $v_{0}(\xi, l)$ and $v_{1}(\xi, l)$ defined by

$$
\begin{aligned}
-\frac{\partial^{2} v_{0}}{\partial \xi^{2}}+b\left(\bar{x}, u_{0}(\bar{x})+v_{0}\right) & =0, \\
-\frac{\partial^{2} v_{1}}{\partial \xi^{2}}+v_{1} b_{u}\left(\bar{x}, u_{0}(\bar{x})+v_{0}\right) & =-\left.\xi \frac{d}{d r} b\left(x, u_{0}(x)+t\right)\right|_{x=\bar{x}, t=v_{0}}-\kappa \frac{\partial v_{0}}{\partial \xi}
\end{aligned}
$$

for $\xi>0$, with the boundary conditions

$$
v_{0}(0, l)=g(\bar{x})-u_{0}(\bar{x}), \quad v_{1}(0, l)=0, \quad v_{0}(\infty, l)=v_{1}(\infty, l)=0 .
$$

Here $x=x(r, l)$ is defined by (2.1),

$$
\bar{x}=\bar{x}(l):=(\varphi(l), \psi(l)),\left.\quad \eta^{-1} \frac{\partial \eta}{\partial r}\right|_{r=0}=\left.\frac{-\kappa}{1-\kappa r}\right|_{r=0}=-\kappa .
$$

Note that for each $l$ the equation for $v_{0}$ is a nonlinear autonomous ODE. Our conditions (A1), (A2) are precisely what is needed to ensure existence and asymptotic properties of $v_{0}$ and $v_{1}$, 8 , 12; see Lemma 2.4.

The following result is a simplified version of [12, Theorem 3].

Theorem 2.2 ([12]). Under hypotheses (A1), (A2), for sufficiently small $\varepsilon$ there exists a solution $u(x)$ of (1.1) in a neighbourhood of the zero-order asymptotic expansion $u_{0}(x)+v_{0}(\xi, l) \omega(x)$. Furthermore, for the first-order asymptotic expansion

$$
u_{\mathrm{as}}(x):=u_{0}(x)+\left[v_{0}(\xi, l)+\varepsilon v_{1}(\xi, l)\right] \omega(x)
$$

we have

$$
\left|F u_{\text {as }}(x)\right| \leq C \varepsilon^{2}, \quad\left|u(x)-u_{\text {as }}(x)\right| \leq C \varepsilon^{2} \quad \text { for all } x \in \bar{\Omega} .
$$

Remark 2.3. The asymptotic expansion from Theorem 2.2 provides an accurate approximation of $u$. Note that in general it is inefficient to compute approximate solutions in this way, because one must solve several auxiliary, possibly nonlinear problems. It is simpler instead to apply a suitable numerical method directly to the original singularly perturbed differential equation.

2.3. Modified asymptotic expansion, sub- and super-solutions. To construct discrete sub- and super-solutions, we shall use the auxiliary function $v(\xi, l ; p)$ that is defined by

$$
-\frac{\partial^{2} v}{\partial \xi^{2}}+b\left(\bar{x}, u_{0}(\bar{x})+v\right)=p v,
$$

with the boundary conditions

$$
\left.v(0, l ; p)=g(\bar{x})-u_{0}(\bar{x}), \quad v(\infty, l ; p)\right)=0 .
$$


Clearly $v(\xi, l ; p)$ is a generalization of $v_{0}(\xi, l)$ from (2.4) and $v(\xi, l ; 0)=v_{0}(\xi, l)$. Furthermore, set

$$
\beta(x ; p):=u_{0}(x)+\left[v(\xi, l ; p)+\varepsilon v_{1}(\xi, l)\right] \omega(x)+C_{0} p,
$$

where $C_{0}$ is a sufficiently small positive constant that will be specified later. Note that (2.10) can be rewritten as

$$
\beta(x ; p)=u_{\text {as }}(x)+w(\xi, l ; p)+C_{0} p, \quad w(\xi, l ; p):=[v(\xi, l ; p)-v(\xi, l ; 0)] \omega(x) .
$$

For some small $p>0$ the functions $\beta(x ;-p)$ and $\beta(x ; p)$ will serve as sub- and super-solutions respectively. The value $p$ in the definition of $v$ and $\beta$ is a small real number that will be chosen later and is typically $o(h)$. Thus for $h$ sufficiently small, by the following lemma, $\beta$ is well defined.

Lemma 2.4. Set $\gamma_{0}^{2}:=\min _{x \in \partial \Omega} b_{u}\left(x, u_{0}(x)\right)>0$. There exists $p_{0} \in\left(0, \gamma_{0}^{2}\right)$ such that for all $|p| \leq p_{0}$ there exist $v_{0}(\xi, l), v_{1}(\xi, l)$, and $v(\xi, l ; p)$ that satisfy (2.4), (2.5), (2.6), (2.8), (2.9). Furthermore, $v_{0} \geq 0$ and $v \geq 0$. Additionally, let $\tilde{\gamma}_{0}^{2}:=\gamma_{0}^{2}-p_{0}$ and $\delta \in\left(0, \tilde{\gamma}_{0}\right)$ be arbitrary but fixed; then there exists a positive constant $C_{\delta}$ such that

$$
\frac{\partial v(\xi, l ; p)}{\partial p} \geq 0
$$

and

$$
\left|\frac{\partial^{k} v(\xi, l ; p)}{\partial \xi^{k}}\right|+\left|\frac{\partial^{k} v_{1}(\xi, l)}{\partial \xi^{k}}\right|+\left|\frac{\partial v(\xi, l ; p)}{\partial p}\right|+\left|\frac{\partial^{2} v(\xi, l ; p)}{\partial p \partial \xi}\right| \leq C_{\delta} e^{-\left(\tilde{\gamma}_{0}-\delta\right) \xi}
$$

for $0 \leq \xi<\infty$, all $l$ and $k=0,1, \ldots, 4$.

Proof. The present lemma is an extended version of [ $[$, Lemma 2.3 and (2.15)]. The estimate for $v_{p \xi}$ is proved similarly to the estimate for $v_{p}$.

Remark 2.5. By (A1), $\gamma_{0}>\gamma$. Choosing $p_{0}$ and $\delta$ sufficiently small, we can make $\tilde{\gamma}_{0}-\delta$ in (2.13) arbitrarily close to $\gamma_{0}$ and $\tilde{\gamma}_{0}-\delta>\gamma$.

Corollary 2.6. We have $(1+\xi)|w|+\left|w_{\xi}\right| \leq C p$, where $w$ is defined in (2.11).

Proof. This follows from estimates (2.13) for $v_{p}$ and $v_{p \xi}$.

Corollary 2.7. We have $\beta(x ; p)=u_{\text {as }}(x)+O(p)=u(x)+O\left(p+\varepsilon^{2}\right)$. Furthermore, if $p \geq 0$, then $\beta(x ;-p) \leq \beta(x ; p)$.

Proof. These two statements follow from Corollary 2.6 combined with (2.7), and (2.10) combined with (2.12), respectively.

Lemma 2.8. We have

$$
F \beta=C_{0} p b_{u}\left(x, u_{0}\right)+\left[1+C_{0} \lambda(x)\right] p v_{0}(\xi, l)+O\left(\varepsilon^{2}+p^{2}\right)
$$

where $\lambda(x):=b_{u u}\left(x, u_{0}+\vartheta v_{0}\right)$ with $\vartheta=\vartheta(x) \in(0,1)$.

Proof. To simplify the presentation, assume that the cut-off function $\omega$ equals 1 in the whole domain $\bar{\Omega}$. Since this is not the case for $\varepsilon \xi>C_{1} / 2$, where the functions $v$ and $v_{1}$ and their derivatives are negligible, this assumption will not influence our conclusions.

Throughout the proof we use the notation $\bar{x}:=(\varphi(l), \psi(l))$ and $\bar{u}_{0}:=u_{0}(\bar{x})$, while $u_{0}, v_{0}, v$, and $w$ are, as usual, abbreviations for $u_{0}(x), v_{0}(\xi, l), v(\xi, l ; p)$, and $w(\xi, l ; p)$ respectively. Note that $v_{0}=v(\xi, l ; 0)$. 
It follows from (2.7) that $\varepsilon^{2} \triangle u_{\text {as }}=b\left(x, u_{\text {as }}\right)+O\left(\varepsilon^{2}\right)$. Furthermore, by (2.2), (2.3), (2.11), we get $\varepsilon^{2} \triangle w=w_{\xi \xi}+O\left(\varepsilon\left|w_{\xi}\right|+\varepsilon^{2}\right)$. Recalling that, by Corollary 2.6, $\left|w_{\xi}\right| \leq C p$, we arrive at

$$
F \beta=-b\left(x, u_{\text {as }}\right)-w_{\xi \xi}+b\left(x, u_{\text {as }}+w+C_{0} p\right)+O\left(p^{2}+\varepsilon^{2}\right) .
$$

Now, by (2.8) combined with $v=v_{0}+w$ and $w=O(p)$, we get

$$
w_{\xi \xi}=\left[b\left(\bar{x}, \bar{u}_{0}+v_{0}+w\right)-b\left(\bar{x}, \bar{u}_{0}+v_{0}\right)\right]-p v_{0}+O\left(p^{2}\right) .
$$

Hence

$$
\begin{aligned}
F \beta= & -b\left(x, u_{\mathrm{as}}\right)-\left[b\left(\bar{x}, \bar{u}_{0}+v_{0}+w\right)-b\left(\bar{x}, \bar{u}_{0}+v_{0}\right)\right]+p v_{0} \\
& +b\left(x, u_{\mathrm{as}}+w+C_{0} p\right)+O\left(p^{2}+\varepsilon^{2}\right) .
\end{aligned}
$$

Introducing the function

$$
\mu(t):=b\left(x, u_{\mathrm{as}}+t\right)-b\left(\bar{x}, \bar{u}_{0}+v_{0}+t\right)
$$

we can rewrite this as

$$
F \beta=\left[b\left(x, u_{\text {as }}+w+C_{0} p\right)-b\left(x, u_{\text {as }}+w\right)\right]+[\mu(w)-\mu(0)]+p v_{0}+O\left(p^{2}+\varepsilon^{2}\right)
$$

Since $u_{\text {as }}=u_{0}+v_{0}+O(\varepsilon)$ and $w=O(p)$, we have

$$
b\left(x, u_{\mathrm{as}}+w+C_{0} p\right)-b\left(x, u_{\mathrm{as}}+w\right)=\left[b_{u}\left(x, u_{0}+v_{0}\right)+O(\varepsilon+p)\right] C_{0} p,
$$

where

$$
b_{u}\left(x, u_{0}+v_{0}\right) C_{0} p=\left[b_{u}\left(x, u_{0}\right)+\lambda(x) v_{0}\right] C_{0} p
$$

Furthermore,

$$
\mu(w)-\mu(0)=w \mu^{\prime}(\bar{\vartheta} w)=\left[b_{u}\left(x, u_{\mathrm{as}}+\bar{\vartheta} w\right)-b_{u}\left(\bar{x}, \bar{u}_{0}+v_{0}+\bar{\vartheta} w\right)\right] w
$$

where $\bar{\vartheta}=\bar{\vartheta}(x) \in(0,1)$. Now by Corollary 2.6, we get

$$
\mu(w)-\mu(0)=O(\varepsilon)[1+\xi]|w|=O(\varepsilon p) .
$$

Combining (2.14)-(2.17), we complete the proof.

Corollary 2.9. There exists $C_{0}>0$ such that for all $|p| \leq p_{0}$ we have

$$
\begin{aligned}
& F \beta \geq \quad C_{0} p \gamma^{2}+O\left(\varepsilon^{2}+p^{2}\right), \quad \text { if } p>0 \text {, } \\
& F \beta \leq-C_{0}|p| \gamma^{2}+O\left(\varepsilon^{2}+p^{2}\right), \quad \text { if } \quad p<0 \text {. }
\end{aligned}
$$

Proof. Recall (A1) and that $v_{0} \geq 0$, by Lemma 2.4. Now choose $0<C_{0} \leq|\lambda(x)|^{-1}$ for all $x$ so that $1+C_{0} \lambda(x) \geq 0$.

Thus if $p>0$ and $C_{0} p \gamma^{2}$ dominates $O\left(\varepsilon^{2}+p^{2}\right)$ in Corollary 2.9, then $F \beta(x ;-p) \leq$ $0 \leq F \beta(x ; p)$, while, by Corollary 2.7, $\beta(x ;-p) \leq \beta(x ; p)$. Such functions $\beta(x ;-p)$ and $\beta(x ; p)$ are called sub- and super-solutions of problem (1.1). 


\section{ANALYSIS OF THE NUMERICAL METHOD}

3.1. Layer-adapted meshes. Introduce a small positive parameter $\sigma$ that will be specified later. Let $\sigma \leq C_{1}$ so that the closed curve $\partial \Omega_{\sigma}$ that is defined by the equation $r=\sigma$ does not intersect itself. Furthermore, let $\Omega_{\sigma}$ be the interior of $\partial \Omega_{\sigma}$. Our problem will be discretized separately in $\Omega_{\sigma}$ and $\Omega \backslash \Omega_{\sigma}$, to which we shall refer as the interior region and the layer region respectively; see Figure 2 ,

The boundary-layer region $\Omega \backslash \Omega_{\sigma}$ is the rectangle $(0, \sigma) \times[0, L]$ in the coordinates $(r, l)$. Hence in this subdomain introduce the tensor-product mesh $\left\{\left(r_{i}, l_{j}\right), i=\right.$ $\left.0, \ldots, N, j=-1, \ldots N_{l}\right\}$, where, as usual, $r_{0}=0, r_{N}=\sigma, l_{0}=0$, and $l_{N_{l}}=L$, while $l_{-1}=l_{N_{l}-1}-L$. Furthermore, let $\left\{l_{j}\right\}$ be a quasiuniform mesh on $[0, L]$, i.e., $C^{-1} h \leq l_{j}-l_{j-1} \leq C h$. The choice of the layer-adapted mesh $\left\{r_{i}\right\}$ on $[0, \sigma]$ is crucial and will be discussed later; see (a), (b). Now assume only that $r_{i}-r_{i-1} \leq h$ and

$$
C^{-1} h^{-1} \leq N \leq C h^{-1}
$$

In the interior region $\Omega_{\sigma}$ introduce a quasiuniform Delaunay triangulation, i.e., the maximum side length of any triangle is at most $h$, the area of any triangle is bounded below by $C h^{2}$, and the sum of the angles opposite to any edge is less than or equal to $\pi$ (while any angle opposite to $\partial \Omega_{\sigma}$ does not exceed $\pi / 2$ ). Then the piecewise linear finite element discretization of the operator $-\triangle$ yields an $M$ matrix.

Furthermore, let the union of all the triangles define a polygonal domain $\Omega_{\sigma}^{h}$ whose boundary vertices lie on $\partial \Omega_{\sigma}$. Note that we do not replace our original domain $\Omega$ by a similar polygonal domain $\Omega^{h}$, since a significant part of the boundary layer would be lost in $\Omega \backslash \Omega^{h}$. We also require that both the interior and layer meshes have the same sets of nodes on $\partial \Omega_{\sigma}$.

We focus on two particular choices of $\left\{r_{i}\right\}$ :

3.1(a) Bakhvalov mesh [1. Set $\sigma:=2 \gamma^{-1} \varepsilon|\ln \varepsilon|$ and define the mesh by

$$
r_{i}:=r([1-\varepsilon] i / N), i=0 \ldots, N, \quad r(t):=-2 \gamma^{-1} \varepsilon \ln (1-t) \text { for } t \in[0,1-\varepsilon] .
$$

3.1(b) Shishkin mesh [16. Set $\sigma=2 \gamma^{-1} \varepsilon \ln N$ and introduce a uniform mesh $\left\{r_{i}\right\}_{i=0}^{N}$ on $[0, \sigma]$, i.e., $r_{i}-r_{i-1}=\sigma / N=2 \gamma^{-1} \varepsilon N^{-1} \ln N$.

Note that if $\varepsilon$ is sufficiently small (recall (A4)) the condition $\sigma \leq C_{1}$ is satisfied and the meshes (a) and (b) are well-defined.

Remark 3.1. If (A4) is not satisfied, but (a) $\sigma \leq C_{1}$ and $\varepsilon \leq 1 / 2$, or (b) $\sigma \leq C_{1}$, the meshes 3.1(a) and 3.1(b) remain well-defined. Otherwise we have $\varepsilon>C$, i.e., our problem is not singularly perturbed. Hence imitating [1, 16], extend the mesh definitions 3.1(a) and 3.1(b) by using the mesh (b) with $\sigma:=C_{1}$. Alternatively, one can simply use linear finite elements on a quasiuniform Delaunay triangulation of the whole domain $\bar{\Omega}$.

Remark 3.2. In the mesh definitions 3.1(a) and 3.1(b) the constant $\gamma$ from (A1) can be replaced by an arbitrary constant $\tilde{\gamma} \in\left(0, \gamma_{0}\right)$, where $\gamma_{0}$ is from Lemma 2.4 see Remark 2.5.

\section{2. $Z$-fields.}

Definition 3.3. An operator $H: \mathbb{R}^{n} \rightarrow \mathbb{R}^{n}$ is a $Z$-field if for all $i \neq j$ the mapping $x_{j} \mapsto\left(H\left(x_{1}, x_{2}, \ldots, x_{n}\right)\right)_{i}$ is a monotonically decreasing function from $\mathbb{R}$ to $\mathbb{R}$ when $x_{1}, \ldots, x_{j-1}, x_{j+1}, \ldots, x_{n}$ are fixed. 
Remark 3.4. If $H$ is differentiable, then $H$ is a $Z$-field if and only if its Jacobian matrix has non-positive off-diagonal entries.

Remark 3.5. An $M$-function [13, Def. 13.5.7] is a particular case of a $Z$-field. To be precise, a mapping $H$ is an $M$-function if $H$ is an inverse-isotone $Z$-field.

We shall use the following unpublished result of Lorenz [9], whose proof is also given in [8].

Lemma 3.6 ([9]). Let $H: \mathbb{R}^{n} \rightarrow \mathbb{R}^{n}$ be continuous and a $Z$-field. Let $r \in \mathbb{R}^{n}$ be given. Assume that there exist $\alpha, \beta \in \mathbb{R}^{n}$ such that $\alpha \leq \beta$ and $H \alpha \leq r \leq H \beta$. (The inequalities are understood to hold true component-wise.) Then the equation $H y=r$ has a solution $y \in \mathbb{R}^{n}$ with $\alpha \leq y \leq \beta$.

Remark 3.7. The functions $\alpha$ and $\beta$ of Lemma 3.6 are called sub- and super-solutions of the discrete problem $H y=r$.

Remark 3.8. Let $X_{1}, X_{2}, \ldots, X_{n}$ be interior points of $\Omega$, while $\bar{X}_{1}, \bar{X}_{2}, \ldots, \bar{X}_{m}$ are on $\partial \Omega$, and let $U \in \mathbb{R}^{n+m}$ be a discrete function defined at these points. Suppose that $F^{h}: \mathbb{R}^{n+m} \rightarrow \mathbb{R}^{n}$ has the form

$$
F^{h} U=\varepsilon^{2} \Lambda U+\left[b\left(X_{i}, U_{i}\right)\right]_{i=1}^{n},
$$

where $\Lambda$ is an $M$-matrix discretization of the operator $-\triangle$. Then the mapping $\left(X_{1}, \ldots, X_{n}, \bar{X}_{1}, \ldots, \bar{X}_{m}\right) \mapsto\left(F^{h} U_{1}, \ldots, F^{h} U_{n}, g\left(\bar{X}_{1}\right), \ldots, g\left(\bar{X}_{m}\right)\right)$ is a $Z$-field.

Remark 3.9. Thus to invoke the theory of $Z$-fields we require the following: (i) an $M$-matrix discretization of $-\triangle$; (ii) the discretization of $b(x, u)$ at any interior mesh point $X_{i}$ involves only $U_{k}$ with $k=i$. Hence we use finite differences in the layer region and lumped mass linear finite elements on Delaunay triangulations in the interior region.

3.3. Discretization in the interior of the boundary-layer region. Recall that $\Omega \backslash \Omega_{\sigma}$ is the rectangle $(0, \sigma) \times[0, L]$ in the coordinates $(r, l)$. Hence rewrite (1.1a) in $(r, l)$ coordinates, by (2.2), and then discretize it using the standard finite differences on the tensor-product mesh $\left\{\left(r_{i}, l_{j}\right)\right\}$ [14. In the interior of $\Omega \backslash \Omega_{\sigma}$, i.e., for $i=1, \ldots, N-1, j=0, \ldots, N_{l}-1$, set

$$
\begin{gathered}
F^{h} U_{i j}:=-\varepsilon^{2} \eta_{i j}^{-1} D_{r}\left[\tilde{\eta}_{i j} D_{r}^{-} U_{i j}\right]-\varepsilon^{2} \zeta_{i j} D_{l}\left[\tilde{\zeta}_{i j} D_{l}^{-} U_{i j}\right]+b\left(x_{i j}, U_{i j}\right)=0, \\
U_{i, N_{l}}=U_{i, 0}, \quad U_{i,-1}=U_{i, N_{l}-1}, \quad U_{0, j}=g\left(x_{0, j}\right) .
\end{gathered}
$$

Here $U_{i j}$ is the discrete computed solution at the mesh node $x_{i j}$,

$$
\begin{aligned}
D_{r}^{-} v_{i j}:=\frac{v_{i j}-v_{i-1, j}}{r_{i}-r_{i-1}}, & D_{r} v_{i j}:=\frac{v_{i+1, j}-v_{i j}}{\left(r_{i+1}-r_{i-1}\right) / 2}, \\
D_{l}^{-} v_{i j}:=\frac{v_{i j}-v_{i, j-1}}{l_{j}-l_{j-1}}, & D_{l} v_{i j}:=\frac{v_{i, j+1}-v_{i j}}{\left(l_{j+1}-l_{j-1}\right) / 2},
\end{aligned}
$$

and

$$
\begin{gathered}
\eta_{i j}:=\eta\left(r_{i}, l_{j}\right), \quad \zeta_{i j}:=\zeta\left(r_{i}, l_{j}\right), \quad x_{i j}:=x\left(r_{i}, l_{j}\right), \\
\tilde{\eta}_{i j}:=\eta\left(r_{i-1 / 2}, l_{j}\right), \quad \tilde{\zeta}_{i j}:=\zeta\left(r_{i}, l_{j-1 / 2}\right) .
\end{gathered}
$$

Remark 3.10. Discretization (3.3) is of type (3.2). 
Lemma 3.11. Let $\beta(x ; p)$ be defined by (2.10), and let the mesh $\left\{r_{i}\right\}_{i=0}^{N}$ be either the Bakhvalov mesh of $\$ 3.1$ (a), or the Shishkin mesh of $\$ 3.1(b)$. Then for all $|p| \leq p_{0}$ at all interior mesh nodes $x_{i j}, i=1, \ldots, N-1, j=0, \ldots, N_{l}-1$ we have

$$
\left|F^{h} \beta_{i j}-F \beta\left(x_{i j}\right)\right| \leq C h^{2}|\ln h|^{m},
$$

where $m=0$ for the Bakhvalov mesh (a) and $m=2$ for the Shishkin mesh (b).

Proof. Note that the term $\varepsilon^{2} \zeta\left(\zeta \beta_{l}\right)_{l}$ in $F \beta$ and its discrete analogue in $F^{h} \beta$ are $O\left(\varepsilon^{2}\right) \leq O\left(h^{2}\right)$, by $(\mathrm{A} 4)$. Now imitate the argument of [8, Lemma 3.3 and $\left.\S 3.4 .2\right]$ and recall (3.1).

3.4. Discretization in the boundary-layer region on the interface boundary $\partial \Omega_{\sigma}$. On the interface boundary $\partial \Omega_{\sigma}$ introduce the fictitious Neumann boundary condition

$$
\frac{\partial u}{\partial r}=\phi(x) \quad \text { for } x \in \partial \Omega_{\sigma},
$$

which will be eliminated when we compile the two discretizations.

For $i=N, j=0, \ldots, N_{l}-1$, following [14, we discretize (1.1a), (2.2) combined with (3.4) as follows:

$$
\begin{gathered}
F_{-}^{h} U_{N j}:=-\varepsilon^{2} \delta_{r}^{2} U_{N j}-\varepsilon^{2} \zeta_{N j} D_{l}\left[\tilde{\zeta}_{N j} D_{l}^{-} U_{N j}\right]+b\left(x_{N j}, U_{N j}\right)=0, \\
U_{N, N_{l}}=U_{N, 0}, \quad U_{N,-1}=U_{N, N_{l}-1}
\end{gathered}
$$

where

$$
\begin{gathered}
\delta_{r}^{2} U_{N j}:=\eta_{N j}^{-1} \frac{\eta_{N j} \phi_{j}-\tilde{\eta}_{N j} D_{r}^{-} U_{N j}}{h_{N} / 2}=\frac{2}{h_{N}} \phi_{j}-\eta_{N j}^{-1} \frac{2}{h_{N}} \tilde{\eta}_{N j} D_{r}^{-} U_{N j}, \\
h_{N}:=r_{N}-r_{N-1}, \quad \phi_{j}:=\phi\left(x_{N j}\right) .
\end{gathered}
$$

The notation $F_{-}^{h}$ reflects the fact that this is the contribution to the discretization on $\partial \Omega_{\sigma}$ from the $r=\sigma^{-}$part of $\Omega$. Furthermore, $F_{-}^{h}$ involves an auxiliary unknown function $\phi$. The actual discretization will be obtained after we discretize the $r=\sigma^{+}$ part of $\Omega$ and then compile the two discretizations by eliminating $\phi$; see 3.6 .

The following lemma presents an auxiliary result that we need to estimate the truncation error near $\partial \Omega_{\sigma}$ in $\Omega_{\sigma}$.

Lemma 3.12. Let $\tilde{\Omega}_{\sigma} \supset \Omega_{\sigma}$ be the interior of the closed curve $r=\sigma-h_{N}$, where $\sigma$ and $\left\{r_{i}\right\}_{i=0}^{N}$ are chosen as in either $33.1(a)$ or $\$ 3.1(b)$. Then for $\beta(x ; p)$ from (2.10) we have $\|\beta\|_{C^{2}\left(\tilde{\Omega}_{\sigma}\right)} \leq C\left(1+h^{2} / \varepsilon^{2}\right)$.

Proof. For $u_{0}$ from (1.2) we have $\left\|u_{0}\right\|_{C^{2}(\Omega)} \leq C$. The derivatives of $v$ and $v_{1}$ (see (2.8), (2.5) ) with respect to $l$ are estimated similarly. To prove the desired estimate for the derivatives of $v$ and $v_{1}$ with respect to $r$, combine estimates (2.13) with $r=\varepsilon \xi$; then recall Remark 2.5 and the values of $\sigma$ and $h_{N}$ for meshes (a) and (b), in particular, (a) $\sigma-h_{N}=2 \gamma^{-1} \varepsilon \ln [1 / O(h)]$ and (b) $h_{N}=O(\varepsilon h|\ln h|)$. Finally, check that $\varepsilon^{-2} e^{-\gamma\left(\sigma-h_{N}\right) / \varepsilon}$ is bounded by $C h^{2} / \varepsilon^{2}$.

Lemma 3.13. Let $\beta(x ; p)$ be defined by (2.10), and let the mesh $\left\{r_{i}\right\}_{i=0}^{N}$ be either the Bakhvalov mesh $₫ 3.1(a)$, or the Shishkin mesh $₫ 3.1(b)$. Then for all $|p| \leq p_{0}$ at all interface-boundary mesh nodes $x_{N j} \in \partial \Omega_{\sigma}$ we have

$$
\left.\left(F_{-}^{h} \beta-F \beta\right)\right|_{x_{N j} \in \partial \Omega_{\sigma}}=\left.\frac{2 \varepsilon^{2}}{h_{N}}\left(\frac{\partial \beta}{\partial r}-\phi\right)\right|_{x_{N j}}+O\left(h^{2}\right) .
$$


Proof. First note that

$$
F_{-}^{h} \beta_{N j}-F \beta\left(x_{N j}\right)=-\varepsilon^{2} \delta_{r}^{2} \beta_{N j}+O\left(\varepsilon^{2}\right)\|\beta\|_{C^{2}\left(\tilde{\Omega}_{\sigma}\right)} .
$$

Now

$$
\delta_{r}^{2} \beta_{N j}=\frac{\phi_{j}-\left.\frac{\partial \beta}{\partial r}\right|_{x_{N j}}}{h_{N} / 2}+\eta_{N j}^{-1} \frac{\left.\eta_{N j} \frac{\partial \beta}{\partial r}\right|_{x_{N j}}-\tilde{\eta}_{N j} D_{r}^{-} \beta_{N j}}{h_{N} / 2},
$$

where the second term on the right-hand side is bounded by $C\|\beta\|_{C^{2}\left(\tilde{\Omega}_{\sigma}\right)}$. Combine these with Lemma 3.12 and (A4), to complete the proof.

3.5. Discretization in the interior part of the domain. Equation (1.1a) in $\Omega_{\sigma}$ combined with the boundary condition (3.4) has the following standard weak form: Find $u \in W_{2}^{1}\left(\Omega_{\sigma}\right)$ such that $\forall v \in W_{2}^{1}\left(\Omega_{\sigma}\right)$ we have

$$
\varepsilon^{2}(\nabla u, \nabla v)+\varepsilon^{2} \oint_{\partial \Omega_{\sigma}} \phi v d s+(b(x, u), v)=0,
$$

where the notation $(\cdot, \cdot)$ is used for the inner product in $L_{2}\left(\Omega_{\sigma}\right)$. Here we used (3.4) rewritten as $\frac{\partial u}{\partial n}=-\phi$ since the outward normal is opposite to the $r$-direction.

Let $S^{h} \subset W_{2}^{1}\left(\Omega_{\sigma}^{h}\right)$ be the standard finite element space of continuous functions that are linear on each of the triangles of our mesh in $\Omega_{\sigma}^{h}$. In $\Omega_{\sigma}^{h}$ define the approximate solution $U \in S^{h}$ by

$$
\varepsilon^{2}\left(\nabla U, \nabla \chi_{i}\right)+\varepsilon^{2} \phi_{i} \oint_{\partial \Omega_{\sigma}^{h}} \chi_{i} d s+b\left(X_{i}, U_{i}\right)\left(1, \chi_{i}\right)=0 \quad \forall \chi_{i} \in S^{h},
$$

where $X_{i}$ is a mesh node in $\bar{\Omega}_{\sigma}^{h}$, while $U_{i}=U\left(X_{i}\right), \phi_{i}=\phi\left(X_{i}\right)$, and $\chi_{i} \in S^{h}$ are the nodal basis functions, i.e., $\chi_{i}\left(X_{j}\right)$ equals 1 if $i=j$ and 0 otherwise. Here we used the lumped mass discretization of both the boundary integral and the integral involving $b$; see Remarks 3.8 and 3.9 .

First, consider (3.8) at interior meshnodes $X_{i}$ of $\Omega_{\sigma}$. For consistency with the finite difference operator $F^{h}$, define

$$
F^{h} U_{i}:=\frac{\varepsilon^{2}}{\left(1, \chi_{i}\right)}\left(\nabla U, \nabla \chi_{i}\right)+b\left(X_{i}, U_{i}\right)=0 \quad \forall X_{i} \in \Omega_{\sigma} .
$$

Remark 3.14. Since we have a Delaunay triangulation, the first term yields an $M$-matrix. Hence (3.9) in the interior mesh nodes of $\Omega_{\sigma}$ is of type (3.2).

Lemma 3.15. Let $\beta=\beta(x ; p)$ be defined by (2.10), and let $\beta^{I} \in S^{h}$ be its piecewise linear interpolant such that $\beta^{I}\left(X_{i}\right)=\beta\left(X_{i}\right)$ at all mesh nodes $X_{i} \in \bar{\Omega}_{\sigma}$. Furthermore, let $\sigma$ be chosen as in either 33.1 (a) or $\$ 3.1(b)$. Then for all $|p| \leq p_{0}$ we have

$$
\left|F^{h} \beta_{i}^{I}-F \beta_{i}\right| \leq C h^{2} \quad \forall X_{i} \in \Omega_{\sigma} .
$$

Proof. We intend to estimate

$$
F^{h} \beta_{i}^{I}-\left.F \beta\right|_{X_{i}}=\frac{\varepsilon^{2}}{\left(1, \chi_{i}\right)}\left(\nabla \beta^{I}, \nabla \chi_{i}\right)+\left.\varepsilon^{2} \triangle \beta\right|_{X_{i}} .
$$

Since $X_{i}$ is an interior node of $\Omega_{\sigma}$, then $\chi_{i}=0$ on $\partial \Omega_{\sigma}^{h}$ and hence we get

$$
\left(\nabla \beta^{I}, \nabla \chi_{i}\right)=\left(\nabla\left[\beta^{I}-\beta\right], \nabla \chi_{i}\right)-\left(\triangle \beta, \chi_{i}\right) .
$$


Combine this with the interpolation error estimate $\left|\nabla\left[\beta^{I}-\beta\right]\right| \leq C h\|\beta\|_{C^{2}\left(\bar{\Omega}_{\sigma}\right)}$ and the standard quasiuniform-mesh properties $\left(1,\left|\nabla \chi_{i}\right|\right) \leq C h$ and $\left(1, \chi_{i}\right) \geq C h^{2}$. Hence

$$
\left|F^{h} \beta_{i}^{I}-F \beta_{i}\right| \leq C \varepsilon^{2}\|\beta\|_{C^{2}\left(\bar{\Omega}_{\sigma}\right)} .
$$

Combining this with Lemma 3.12 and (A4), we complete the proof.

3.6. Discretization on the interface boundary $\partial \Omega_{\sigma}$. Now consider (3.8) at mesh nodes $X_{j}$ on the interface boundary $\partial \Omega_{\sigma}$ and define

$$
F_{+}^{h} U_{j}:=\frac{\varepsilon^{2}}{\left(1, \chi_{j}\right)}\left(\nabla U, \nabla \chi_{j}\right)+\frac{\varepsilon^{2} a_{j}}{h} \phi_{j}+b\left(X_{j}, U_{j}\right)=0 \quad \forall X_{j} \in \partial \Omega_{\sigma},
$$

where

$$
a_{j}:=\frac{h}{\left(1, \chi_{j}\right)} \oint_{\partial \Omega_{\sigma}^{h}} \chi_{j} d s, \quad 0<C^{-1}<a_{j}<C .
$$

The second relation in (3.12) holds true for all $j$ since in $\Omega_{\sigma}$ our triangulation is quasiuniform.

Lemma 3.16. Under the conditions of Lemma 3.15, for all $|p| \leq p_{0}$ we have

$$
F_{+}^{h} \beta_{j}^{I}-F \beta_{j}=-\left.a_{j} \frac{\varepsilon^{2}}{h}\left(\frac{\partial \beta}{\partial r}-\phi\right)\right|_{X_{j}}+O\left(h^{2}\right) \quad \forall X_{j} \in \partial \Omega_{\sigma} .
$$

Proof. We intend to estimate

$$
F_{+}^{h} \beta_{j}^{I}-\left.F \beta\right|_{X_{j}}=\frac{\varepsilon^{2}}{\left(1, \chi_{j}\right)}\left(\nabla \beta^{I}, \nabla \chi_{j}\right)+\varepsilon^{2} \frac{a_{j}}{h} \phi_{j}+\left.\varepsilon^{2} \triangle \beta\right|_{X_{j}} .
$$

Imitate the argument of Lemma 3.15 with the difference that now $\chi_{j}$ does not vanish on $\partial \Omega_{\sigma}^{h}$. This results in additional terms that involve integrals along $\partial \Omega_{\sigma}^{h}$. Instead of (3.10) we have

$$
\left(\nabla \beta^{I}, \nabla \chi_{j}\right)=\left(\nabla\left[\beta^{I}-\beta\right], \nabla \chi_{j}\right)-\left(\triangle \beta, \chi_{j}\right)+\oint_{\partial \Omega_{\sigma}^{h}} \chi_{j} \frac{\partial \beta}{\partial n} d s .
$$

Here $\frac{\partial \beta}{\partial n}$ is the derivative in the direction of the outward normal computed at points within $O(h)$-distance from $X_{j}$, for which we have

$$
\frac{\partial \beta}{\partial n}=-\left.\frac{\partial \beta}{\partial r}\right|_{X_{j}}+O(h)\|\beta\|_{C^{2}\left(\bar{\Omega}_{\sigma}\right)} .
$$

To get this, we also noted that due to smoothness of $\Omega_{\sigma}$, the directions of $-r$ at $X_{j}$ and the normal $n$ to $\partial \Omega_{\sigma}^{h}$ in the $O(h)$-neighbourhood of $X_{j}$ differ by at most $O(h)$. To complete the proof, use $\oint_{\partial \Omega_{\alpha}^{h}} \chi_{j} d s \leq C h$, Lemma 3.12, and (A4).

Now we compile the discretizations $F_{-}^{h}(3.5)$, (3.6) and $F_{+}^{h}$ (3.11), (3.12) by eliminating the auxiliary unknown function $\phi$ and obtaining the actual discretization on the interface boundary $\partial \Omega_{\sigma}$ :

$$
F^{h} U_{j}:=\frac{\left(h_{N} / 2\right) F_{-}^{h} U_{j}+\left(h / a_{j}\right) F_{+}^{h} U_{j}}{h_{N} / 2+h / a_{j}} \quad \forall X_{j} \in \partial \Omega_{\sigma} .
$$

Remark 3.17. By (3.5), (3.6), (3.11), (3.12), the discretization (3.14) is of type (3.2). 
Lemma 3.18. Under the conditions of Lemma 3.13, for $F^{h}$ of (3.14) we have

$$
\left|F^{h} \beta_{j}-F \beta_{j}\right| \leq C h^{2} \quad \forall X_{j} \in \partial \Omega_{\sigma} .
$$

Proof. Add (3.7) multiplied by $\left(h_{N} / 2\right)$ to (3.13) multiplied by $\left(h / a_{j}\right)$ and divide the result by $\left(h_{N} / 2+h / a_{j}\right)$.

\subsection{Existence and accuracy, discrete sub- and super-solutions.}

Lemma 3.19. Set $\bar{p}:=C_{2} h^{2}|\ln h|^{m}$, where $m=0$ for the Bakhvalov mesh of $\S 3.1$ (a) and $m=2$ for the Shishkin mesh of $\S 3.1(b)$, while $C_{2}>0$ is a sufficiently large constant. Let $\beta(x ; p)$ be defined by (2.10) and $h \leq C_{3}$, where $C_{3}>0$ is sufficiently small. Then $\beta\left(X_{i} ;-\bar{p}\right)$ and $\beta\left(X_{i} ; \bar{p}\right)$ are discrete sub- and supers-solutions (see Remark 3.7).

Proof. Combine Lemmas 3.11 , 3.15 and 3.18 and then choose $C_{2}$ sufficiently large to get $\left|F^{h} \beta-F \beta\right| \leq C h^{2}|\ln h|^{m} \leq \bar{p} / 2$ for all $|p| \leq p_{0}$. Now, by Corollary 2.9 and (A4), if $C_{2}$ is sufficiently large and $h$ is sufficiently small, we have $F \beta(x ;-\bar{p}) \leq-\bar{p} / 2$ and $F \beta(x ; \bar{p}) \geq \bar{p} / 2$. Hence $F^{h} \beta\left(X_{i} ;-\bar{p}\right) \leq 0$ and $F^{h} \beta\left(X_{i} ; \bar{p}\right) \geq 0$. Furthermore, by Corollary 2.7. we have $\beta\left(X_{i} ;-\bar{p}\right) \leq \beta\left(X_{i}, \bar{p}\right)$. We are done; see Remark 3.7 .

The following theorem states existence and $\varepsilon$-uniform accuracy of multiple discrete solutions.

Theorem 3.20. Let the mesh $\left\{r_{i}\right\}_{i=0}^{N}$ be either the Bakhvalov mesh of $\$ 3.1$ (a), or the Shishkin mesh of 33.1 (b). There exists a discrete solution $U$ of (3.3), (3.9), (3.14) such that for $h$ sufficiently small,

$$
\left|U\left(X_{i}\right)-u\left(X_{i}\right)\right| \leq C h^{2}|\ln h|^{m} \quad \forall \text { mesh nodes } X_{i} \in \bar{\Omega},
$$

where $m=0$ for the Bakhvalov mesh (a) and $m=2$ for the Shishkin mesh (b).

Proof. The discrete operator $F^{h}$ is a $Z$-field; see Remarks 3.8, 3.10, 3.14, 3.17. By Lemma 3.19, $\beta\left(X_{i} ;-\bar{p}\right)$ and $\beta\left(X_{i} ; \bar{p}\right)$ are discrete sub- and super-solutions. Hence, by Lemma 3.6, there exists a discrete solution $U$ such that $\beta\left(X_{i} ;-\bar{p}\right) \leq U\left(X_{i}\right) \leq$ $\beta\left(X_{i} ; \bar{p}\right)$. Furthermore, by Corollary 2.7, $U\left(X_{i}\right)=u\left(X_{i}\right)+O\left(\bar{p}+\varepsilon^{2}\right)$. Finally, recall (A4) to get the desired error estimate.

Remark 3.21. Theorem 3.20 is obtained under the assumption (A4) that $\varepsilon \leq C h$. If $\varepsilon>C h$, in accordance with the error estimates of Schatz and Wahlbin [15, we expect a slightly modified version of (3.15):

$$
|u-U| \leq C\left[h^{2} \ln (1+\varepsilon / h)+h^{2}|\ln h|^{m}\right] .
$$

The above estimate differs from (3.15) by the term $h^{2} \ln (1+\varepsilon / h)$, which we find in the local maximum-norm error estimate [15, (1.8)]. We expect this local estimate to work in the interior region, where a quasiuniform Delaunay triangulation is used, with the pollution effect being $O\left(h^{2}|\ln h|^{m}\right)$ due to layer-adapted mesh refinement. Note also that while $h^{2} \ln (1+\varepsilon / h)$ is $O\left(h^{2}\right)$ when $\varepsilon \leq C h$, it gradually increases to the classical maximum-norm finite-element error bound $O\left(h^{2}|\ln h|\right)$ as $\varepsilon$ gradually increases to $O(1)$. 


\section{NUMERICAL RESULTS}

Our model problem is (1.1) in the domain $\Omega$ (see Figure 2) whose boundary $\partial \Omega$ is parameterized by $x_{1}=\varphi(l):=R \cos \theta$ and $x_{2}=\psi(l):=R \sin \theta$, where $l \in[0,2 \pi]$,

$$
R=R(l)=0.4+\cos ^{2}(l / 2), \quad \theta=\theta(l)=l+e^{(l-5) / 2} \sin (l / 2) \sin l .
$$

We use

$$
b(x, u)=\left(u-\bar{u}_{0}(x)\right) u\left(u+\bar{u}_{0}(x)\right), \quad \bar{u}_{0}(x)=x_{1}^{2}+x_{1}+1 .
$$

Here $\pm \bar{u}_{0}(x)$ are two stable solutions and 0 is an unstable solution of the corresponding reduced problem. The boundary condition $g(x)=\left(x_{1}-x_{1}^{2}\right) / 3$ satisfies (A2) for both $\pm \bar{u}_{0}$; see Figure 1 . We present numerical results only for the solution $u$ near $\bar{u}_{0}$; see Figure 1 (left); the results for the solution near $-\bar{u}_{0}$ are similar.

To solve the discrete nonlinear problem we used Newton's method. Tables 1, 2 give numerical results for the Bakhvalov and Shishkin meshes with $\gamma:=0.8 \gamma_{0}$, where $\gamma_{0}=3 \sqrt{2} / 4$. They show rates of convergence and maximum nodal errors computed as described in [8, $\$ 4]$.

Our results confirm the sharpness of the bound of Theorem 3.20 .

TABle 1. Bakhvalov mesh. Computational rates $r$ in $\left(N^{-1}\right)^{r}$ and maximum nodal errors

\begin{tabular}{cccc}
\hline$N$ & $\varepsilon=10^{-2}$ & $\varepsilon=10^{-4}$ & $\varepsilon=10^{-8}$ \\
\hline 32 & 2.010 & 2.011 & 2.011 \\
64 & 1.995 & 1.997 & 1.997 \\
128 & 1.995 & 2.001 & 2.001 \\
\hline 32 & $3.745 \mathrm{e}-3$ & $3.842 \mathrm{e}-3$ & $3.843 \mathrm{e}-3$ \\
64 & $9.296 \mathrm{e}-4$ & $9.534 \mathrm{e}-4$ & $9.536 \mathrm{e}-4$ \\
128 & $2.333 \mathrm{e}-4$ & $2.388 \mathrm{e}-4$ & $2.388 \mathrm{e}-4$ \\
256 & $5.854 \mathrm{e}-5$ & $5.967 \mathrm{e}-5$ & $5.968 \mathrm{e}-5$ \\
\hline
\end{tabular}

TABle 2. Shishkin mesh. Computational rates $r$ in $\left(N^{-1} \ln N\right)^{r}$ and maximum nodal errors

\begin{tabular}{cccc}
\hline$N$ & $\varepsilon=10^{-2}$ & $\varepsilon=10^{-4}$ & $\varepsilon=10^{-8}$ \\
\hline 32 & 2.131 & 2.137 & 2.137 \\
64 & 2.210 & 2.045 & 2.045 \\
128 & 2.465 & 2.009 & 2.009 \\
\hline 32 & $3.915 \mathrm{e}-2$ & $3.947 \mathrm{e}-2$ & $3.948 \mathrm{e}-2$ \\
64 & $1.318 \mathrm{e}-2$ & $1.325 \mathrm{e}-2$ & $1.325 \mathrm{e}-2$ \\
128 & $4.004 \mathrm{e}-3$ & $4.400 \mathrm{e}-3$ & $4.401 \mathrm{e}-3$ \\
256 & $1.008 \mathrm{e}-3$ & $1.430 \mathrm{e}-3$ & $1.430 \mathrm{e}-3$ \\
\hline
\end{tabular}




\section{Appendix A. Proof of Lemma 2.1}

Proof. The new coordinates are clearly orthogonal since

$$
\left[\begin{array}{ll}
x_{1, r} & x_{1, l} \\
x_{2, r} & x_{2, l}
\end{array}\right]=\left[\begin{array}{ll}
n_{1} & \varphi^{\prime}+r n_{1}^{\prime} \\
n_{2} & \psi^{\prime}+r n_{2}^{\prime}
\end{array}\right]
$$

and the vector columns here are orthogonal. Hence for $\triangle u$ we have

$$
\triangle u=\frac{1}{H_{r} H_{l}}\left[\frac{\partial}{\partial r}\left(\frac{H_{l}}{H_{r}} \frac{\partial u}{\partial r}\right)+\frac{\partial}{\partial l}\left(\frac{H_{r}}{H_{l}} \frac{\partial u}{\partial l}\right)\right],
$$

where $H_{r}$ and $H_{l}$ are the Lamé coefficients:

$$
H_{r}:=\sqrt{x_{1, r}^{2}+x_{2, r}^{2}}=1, \quad H_{l}:=\sqrt{x_{1, l}^{2}+x_{2, l}^{2}}=(1-\kappa r) \tau .
$$

Here $H_{r}=1$ because $\left(n_{1}, n_{2}\right)$ is a unit vector. To prove that $H_{l}=(1-\kappa r) \tau$, we check that

$$
(1-\kappa r)^{2} \tau^{2}=\tau^{2}\left[1-2 \kappa r+\kappa^{2} r^{2}\right]
$$

equals

$$
x_{1, l}^{2}+x_{2, l}^{2}=\left(\varphi^{\prime}+r n_{1}^{\prime}\right)^{2}+\left(\psi^{\prime}+r n_{2}^{\prime}\right)^{2}=\tau^{2}+2 r\left(\varphi^{\prime} n_{1}^{\prime}+\psi^{\prime} n_{2}^{\prime}\right)+r^{2}\left(n_{1}^{\prime 2}+n_{2}^{\prime 2}\right) .
$$

It is suffices to show that

$$
-\tau^{2} \kappa=\varphi^{\prime} n_{1}^{\prime}+\psi^{\prime} n_{2}^{\prime} \quad \text { and } \quad \tau^{2} \kappa^{2}=n_{1}^{\prime 2}+n_{2}^{\prime 2} .
$$

The first relation here follows from

$$
\varphi^{\prime} n_{1}^{\prime}+\psi^{\prime} n_{2}^{\prime}=\varphi^{\prime}\left(-\psi^{\prime} / \tau\right)^{\prime}+\psi^{\prime}\left(\varphi^{\prime} / \tau\right)^{\prime}=-\left(\kappa \tau^{3}\right) / \tau+\left(-\varphi^{\prime} \psi^{\prime}+\psi^{\prime} \varphi^{\prime}\right)(1 / \tau)^{\prime} .
$$

To prove the second relation in A.3), we square both sides of the first and get $\tau^{4} \kappa^{2}=\left(\varphi^{\prime} n_{1}^{\prime}+\psi^{\prime} n_{2}^{\prime}\right)^{2}=\left(\tau^{2}-\psi^{\prime 2}\right) n_{1}^{\prime 2}+\left(\tau^{2}-\varphi^{\prime 2}\right) n_{2}^{\prime 2}+2 \varphi^{\prime} n_{1}^{\prime} \psi^{\prime} n_{2}^{\prime}=\tau^{2}\left(n_{1}^{\prime 2}+n_{2}^{\prime 2}\right)-R^{2}$,

where

$$
\begin{aligned}
R & :=-\psi^{\prime} n_{1}^{\prime}+\varphi^{\prime} n_{2}^{\prime}=\psi^{\prime}\left(\psi^{\prime} / \tau\right)^{\prime}+\varphi^{\prime}\left(\varphi^{\prime} / \tau\right)^{\prime}=\left(\psi^{\prime 2}+\varphi^{\prime 2}\right)^{\prime} /(2 \tau)-\left(\psi^{2}+\varphi^{\prime 2}\right) \tau^{\prime} / \tau^{2} \\
& =\left(\tau^{2}\right)^{\prime} /(2 \tau)-\tau^{\prime}=0 .
\end{aligned}
$$

Finally, (2.2) follows immediately from (A.1), (A.2).

\section{REFERENCES}

[1] N. S. Bakhvalov, On the optimization of methods for solving boundary value problems with boundary layers, Zh. Vychisl. Mat. Mat. Fis., 9 (1969) 841-859 (in Russian). MR0255066 (40:8273)

[2] I.A. Blatov, Galerkin finite-element method for elliptic quasilinear singularly perturbed boundary problems. I, (Russian) Differ. Uravn., 28 (1992), 1168-1177; translation in Differ. Eqs., 28 (1992), 931-940. MR1201213 (94a:65056)

[3] I.A. Blatov, Galerkin finite element method for elliptic quasilinear singularly perturbed boundary problems. II, (Russian) Differ. Uravn., 28 (1992), 1799-1810; translation in Differ. Eqs., 28 (1992), 1469-1477. MR1208410 (94c:65130)

[4] C. Clavero, J. L. Gracia, E. O'Riordan, A parameter robust numerical method for a two dimensional reaction-diffusion problem, Math. Comp., 74 (2005), 1743-1758. MR 2164094 (2006e:65192)

[5] C.M. D'Annunzio, Numerical analysis of a singular perturbation problem with multiple solutions, Ph.D. Dissertation, University of Maryland at College Park, 1986 (unpublished).

[6] P.C. Fife, Semilinear elliptic boundary value problems with small parameters, Arch. Rational Mech. Anal., 52 (1973), 205-232. MR0374665 (51:10863)

[7] P. Grindrod, Patterns and Waves: The theory and applications of reaction-diffusion equations, Clarendon Press, Oxford, 1991. MR.1136256 (92k:35145) 
[8] N. Kopteva and M. Stynes, Numerical analysis of a singularly perturbed nonlinear reactiondiffusion problem with multiple solutions, Appl. Numer. Math., 51 (2004), 273-288. MR2091404 (2005e:65097)

[9] J. Lorenz, Nonlinear singular perturbation problems and the Enquist-Osher scheme, Report 8115, Mathematical Institute, Catholic Univerity of Nijmegen, 1981 (unpublished).

[10] J.M. Melenk, hp-finite element methods for singular perturbations, Springer, 2002. MR1939620 (2003i:65108)

[11] J.D. Murray, Mathematical Biology. Second corrected edition, Springer-Verlag, Berlin, 1993. MR.1239892 (94j:92002)

[12] N.N. Nefedov, The method of differential inequalities for some classes of nonlinear singularly perturbed problems with internal layers, (Russian) Differ. Uravn., 31 (1995), 1142-1149; translation in Differ. Eqs., 31 (1995), 1077-1085. MR1429769 (97m:35018)

[13] J.M. Ortega and W.C. Rheinboldt, Iterative Solution of Nonlinear Equations in Several Variables, Academic Press, New York, 1970. MR.0273810 (42:8686)

[14] A.A. Samarski, Theory of Difference Schemes, Nauka, Moscow, 1989 (in Russian). MR.1196231 (93g:65004)

[15] A.H. Schatz and L.B. Wahlbin, On the finite element method for singularly perturbed reaction-diffusion problems in two and one dimensions, Math. Comp., 40 (1983), 47-89. MR0679434 (84c:65137)

[16] G.I. SHISHKIN, Grid approximation of singularly perturbed elliptic and parabolic equations, Ur. O. Ran, Ekaterinburg, 1992 (in Russian).

[17] G. Sun and M. Stynes, A uniformly convergent method for a singularly perturbed semilinear reaction-diffusion problem with multiple solutions, Math. Comp., 65 (1996), 1085-1109. MR.1351205 (96j:65067)

[18] A.B. Vasil'eva, V.F. Butuzov and L.V. Kalachev, The boundary function method for singular perturbation problems. SIAM Studies in Applied Mathematics, 14, Society for Industrial and Applied Mathematics (SIAM), Philadelphia, PA, 1995. MR.1316892 (96a:34119)

[19] M.I. Višik and L.A. Lyusternik, Regular degeneration and boundary layer for linear differential equations with small parameter, (Russian), Uspehi Mat. Nauk, 12 (1957), 3-122. MR0096041(20:2539)

Department of Mathematics and Statistics, University of Limerick, Limerick, Ireland E-mail address: natalia.kopteva@ul.ie 